\title{
Application of Virtualization Technologies in Novel Industrial Automation: Catalyst or Show-Stopper?
}

\author{
Michael Gundall*, Daniel Reti*, and Hans D. Schotten*† \\ ${ }^{*}$ German Research Center for Artificial Intelligence GmbH (DFKI), \\ Kaiserslautern, Germany \\ ${ }^{\dagger}$ Department of Electrical and Computer Engineering, Technische Universität Kaiserslautern, \\ Kaiserslautern, Germany \\ Email: $\{$ michael.gundall, daniel.reti, hans_dieter.schotten $\} @$ dfki.de
}

\begin{abstract}
Industry 4.0 describes an adaptive and changeable production, where its factory cells have to be reconfigured at very short intervals, e.g. after each workpiece. Furthermore, this scenario cannot be realized with traditional devices, such as programmable logic controllers. Here the use of well-proven technologies of the information technology are conquering the production hall (IT-OT convergence). Therefore, both virtualization and novel communication technologies are being introduced in the field of industrial automation. In addition, these technologies are seen as the key for facilitating various emerging use cases. However, it is not yet clear whether each of the dedicated hardware and software components, which have been developed for specific control tasks and have performed well over decades, can be upgraded without major adjustments.

In this paper, we examine the opportunities and challenges of hardware and operating system-level virtualization based on the stringent requirements imposed by industrial applications. For that purpose, benchmarks for different virtualization technologies are set by determining their computational and networking overhead, configuration effort, accessibility, scalability, and security.
\end{abstract}

Index Terms-Smart Manufacturing, Virtualization Technologies, Container, Virtual Machines, Automation Systems, Networking, Performance Benchmarking, Industrial Security

\section{INTRODUCTION}

The industrial Internet of Things (IIoT), in conjunction with industrial cyber-physical systems (ICPSs) are seen as the basis for the realization of a smart manufacturing, twhich introduces novel use cases that bring new challenges for both communication and automation systems. These can be mobility requirements, the application of computing-intensive algorithms, such as for machine learning (ML) or artificial intelligence (AI), and a highly flexible reconfiguration of manufacturing systems or entire factories. Here, a reconfiguration or even redeployment of industrial automation systems at very short intervals, e.g. before each workpiece, is conceivable, while recent industrial installations are typically based on dedicated

This research was supported by the German Federal Ministry of Education and Research (BMBF) within the Tactile Internet 4.0 (TACNET 4.0) and SCRATCh projects under grant numbers 16KIS0712K and 01IS18062E. The SCRATCh project is part of the ITEA 3 cluster of the European research program EUREKA. The responsibility for this publication lies with the authors. This is a preprint of a work accepted but not yet published at the IEEE 18th International Conference on Industrial Informatics (INDIN). Please cite as: M. Gundall, D Reti, and H.D. Schotten: "Application of Virtualization Technologies in Novel Industrial Automation: Catalyst or Show-Stopper?". In: 2020 18th International Conference on Industrial Informatics (INDIN), IEEE, 2020. hardware controllers, such as programmable logic controllers (PLCs), that cannot provide these capabilities [1]. In order to address these challenges, virtualization is a suitable approach. The virtualization of industrial automation systems enables the application of concepts that are already well-known in the field of information technology (IT). Since most of these concepts cannot be applied "out of the box", the question arises how these concepts can be adopted for industrial purposes.

Furthermore, the convergence between IT and operational technology (OT), and the connection to the IIoT makes security an important topic, which until now has not had much relevance in the industrial communication technology (ICT)[2]. This was not intended as these systems were inherently secure against network intrusion, as there were no connections to the Internet and only few wireless connections. As security mechanisms should not compromise the performance of the system, in many cases existing solutions cannot be used. Therefore, security has to be considered already in an early phase of new installations and has been a concern in our investigations. In this paper, the following contributions can be found:

- Identification of challenges and opportunities imposed by industrial use cases and the most important requirements.

- Evaluation of different virtualization technologies and configurations based on the identified requirements.

Therefore, the paper is structured as follows: Sec. [I] motivates our work on this topic, while Sec. III provides insights into challenges in industrial automation. An overview of virtualization technologies is given in Sec. IV] followed by a benchmarking of the proposed technologies and specific configurations (Sec. $\mathrm{V}$. Finally, a conclusion of the paper is given in Sec.VI

\section{BACKGROUND}

In order to allow a smart manufacturing, a multitude of novel use cases have to be realized. Due to the mobility requirements of devices and process equipment, the number of wireless connections between devices in the industrial landscape will increase rapidly. Therefore, the authors in [3] describe a variety of emerging use cases that rely on wireless communications. Here, it must be taken into account that these devices often only have limited resources available. For instance, if ML, AI, or advanced control algorithms shall be applied, the computing 
power of these resource constrained devices is not sufficient. To overcome this issue, the application of computation offloading is a suitable approach. In this concept, a cloud service performs the calculation of the complex algorithm and only transfers the output values to the resource constrained devices [4]. Another point is the limited battery life time of mobile applications, such as mobile robots or drones for industrial inspection. If the complexity of the algorithm to be performed exceeds a certain level, it is beneficial not to execute it on the mobile device itself, but to offload it to a cloud instance [5]. Therefore a longer battery life time of the device and an increase of its availability and productivity can be achieved.

Another area where the application of virtualization technologies is advantageous is the virtualization of industrial automation systems. Here, a number of benefits can be achieved by applying these approaches. One of these is the access to high performance computer systems that enable the previously mentioned gains of computing offloading. On the other hand the virtualization of devices and applications offers a further level of abstraction with which both their flexibility and their availability can be enhanced. In order to evaluate this, [6] have evaluated a virtualized PLC based on virtual machines (VMs) that are running on Windows. By using this setup a variety of applications can already be realized. However, they limited the range to applications that do not have stringent requirements on latency and determinism, the so-called soft real-time (RT) applications. In this context, the introduction of container-based virtualization can offer benefits. For this reason, [7] proposes a container-based architecture for flexible industrial control applications and provide performance benchmarks for containers running on a Raspberry Pi 2 with and without additional load. However, they assume an interval of $1 \mathrm{~ms}$ for the cyclic execution of an application, which may be too high for some industrial applications. Therefore we vary the interval within the limits of $1 \mathrm{~ms}-1 \mu \mathrm{s}$. In addition, [8] investigated how the determinism of applications running in and outside a container is related to a given RT priority and measured the handling latency of the virtual network interface of containers for the standard network drivers, thus determining the overhead of virtualization on the network interface. As it can be advantageous for industrial applications to use a network driver other than the standard one, we have included all of them in our investigations.

\section{IndUSTRIAL AUtomation}

Very characteristic for industrial applications are the high requirements on the cycle time, determinism, and availability, that vary tremendously from applications on the office floor. In this area, closed loop motion control is the most demanding use case group [3]. This includes use cases such as machine tools, packaging, and printing machines, that typically require a maximum cycle time of $0.5-2 \mathrm{~ms}$, a synchronicity of $1-5 \mu \mathrm{s}$ and a maximum failure of less than one minute per year.

Therefore, the IEC 61131-3 describes the functionality and programming of PLCs. PLCs are sophisticated hardware controllers, that usually provide low processing times and a high availability. In addition to the processing time of the PLC, the cycle time also includes the duration of the data transmission from the sensors to the PLC and the reception of the output values by the actuator. Consequently, the underlying communication system also has to fulfil these demands. In order to reliably satisfy the requirements of all use case types, very heterogeneous communication protocols, the so-called Industrial Ethernet (IE) protocols, were developed. These protocols are typically divided into the following RT classes [9]:

- RT class A: $t_{\text {cycle }}<100 \mathrm{~ms}$,

- RT class B: $t_{\text {cycle }}<10 \mathrm{~ms}$, and

- RT class C: $t_{\text {cycle }}<1 \mathrm{~ms}$.

In particular, protocols targeting RT class $\mathrm{C}$ are often not based on the Internet Protocol (IP) layer, which represents the network layer (layer 3 (L3)) of the Open Systems Interconnection (OSI) model, but build directly on top of the Media Access Control (MAC) layer [9]. This corresponds to the data link layer (layer 2 (L2)) of the OSI model. This method saves the overhead of UDP/IP or TCP/IP header, which has several advantages. Firstly, more user data can be transmitted within the same packet size or the packet size can be reduced. Consequently, the number of devices can be increased and the data packets can be processed faster. For this reason, novel communication technologies and communication protocols such as time-sensitive networking (TSN) and the Open Platform Communications Unified Architecture (OPC UA) standard with its L2-based Publish/Subscribe pattern for RT traffic are also based on these benefits [10]. The possibility of L2 communication is therefore a prerequisite if use cases of RT class $C$ are to be addressed with the virtualized industrial automation system.

In the case of mobile use cases, high demands arise on wireless communications that cannot be met by state of the art solutions. Here, 5th generation wireless communication system (5G) is seen as a highly promising candidate. In order to integrate $5 \mathrm{G}$ in the industrial landscape, [11] proposed a concept that foresees to present the $5 \mathrm{G}$ system to the TSN system like any other TSN-aware bridge.

With the emerging interconnectivity of OT, the attack surface is growing as well, requiring security considerations to be part of the infrastructure design process [3]. In order to evaluate the security of different network configurations a threat model for a generalized industrial automation network topology was made (see Fig. 1). The network consists of three factory halls, a factory cloud, and a demilitarized zone (DMZ) that are connected over a router. In the DMZ servers are reachable from the internet and only have a limited connection to the internal network. In each factory hall an edge server is located where multiple virtualized industrial automation systems run on. Within a factory hall all devices are connected over a L2 bridge. Four types of attackers are defined depending on their location in the network. As shown in Fig. 1 the weakest attacker, here defined as D, can only reach the DMZ of the network from the internet, attacker $\mathrm{C}$ is located in the factory network but on a different subnet. This attacker can enumerate the network and try to pivot to different hosts. Attacker B has compromised a virtualized industrial automation system on the edge server and aims, for example, to escape the container restrictions and to laterally move to other hosts. Attacker A has already compromised the edge server and can, with the respective privileges, control the running container 


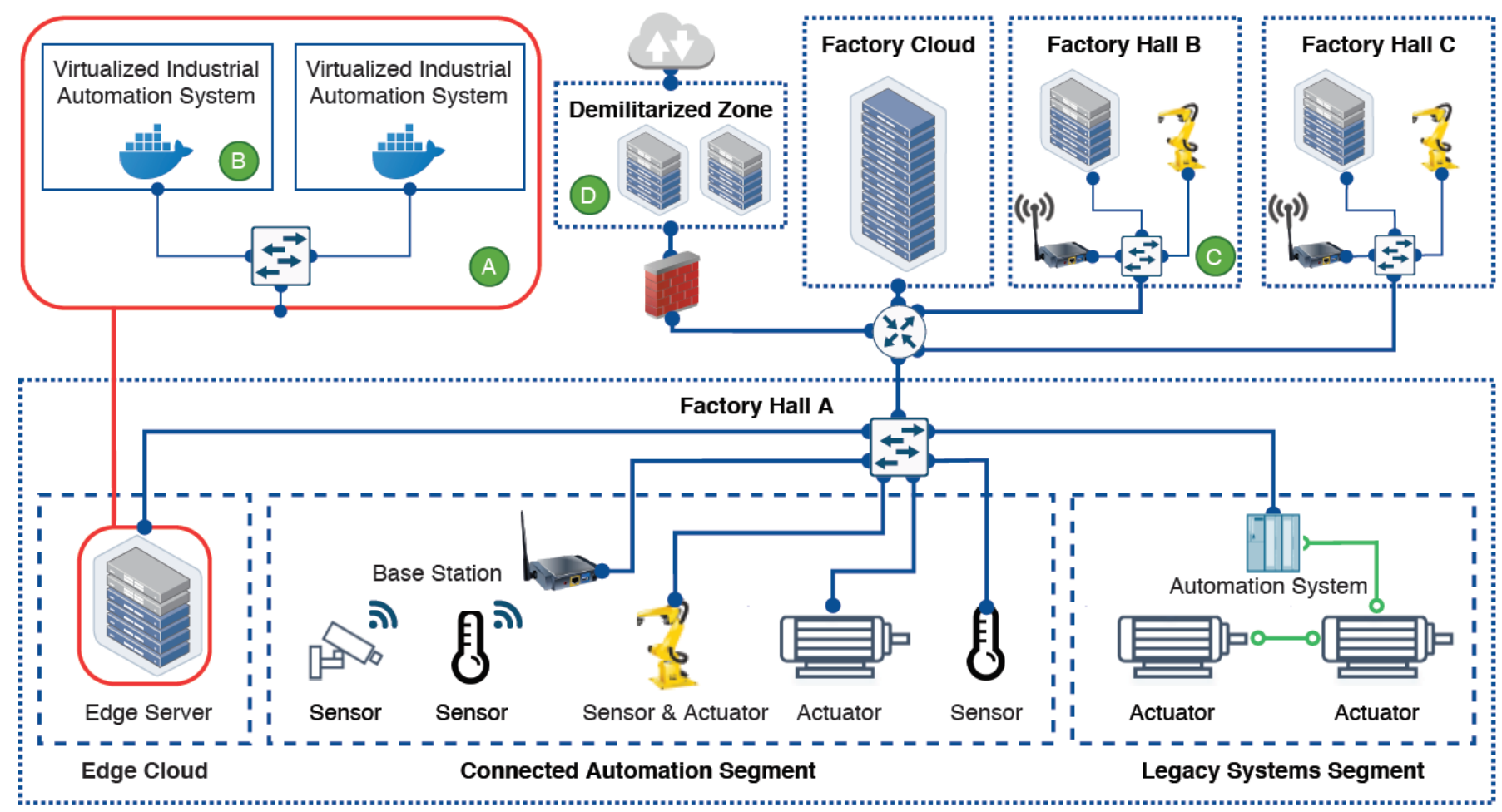

Figure 1. Industrial automation network including possible attacker locations on a generalized network threat model.

daemon and therefore change the containers configuration and connect to them. From here the attacker could steal information, cause outages (denial of service) or manipulate sensor and actuator data [12] to damage the machinery and thereby pose fatal risk to the factory staff.

\section{Virtualization CONCEPTS}

\begin{tabular}{|c|c|c|c|}
\hline VM & VM & & \\
\hline App A & App B & \multirow{2}{*}{ Container } & \multirow{2}{*}{ Container } \\
\hline Bins/Libs & Bins/Libs & & \\
\hline Guest OS & Guest OS & App A & App B \\
\hline Virtual HW & Virtual HW & Bins/Libs & Bins/Libs \\
\hline \multicolumn{2}{|c|}{ Hypervisor } & \multicolumn{2}{|c|}{ Docker Engine } \\
\hline \multicolumn{2}{|c|}{ Host OS } & \multicolumn{2}{|c|}{ Host OS } \\
\hline \multicolumn{2}{|c|}{ Bare-Metal Server } & \multicolumn{2}{|c|}{ Bare-Metal Server or VM } \\
\hline
\end{tabular}

Figure 2. Comparison of abstraction layers between VMs and containers.

Virtualization refers to the replacement of physical resources with virtual replicas. In enterprise environments the main virtualization technology is server virtualization, where traditionally a software called hypervisor may run multiple virtual guest machines on the same physical hardware of the host machine. In the recent decade operating system (OS)-level virtualization gained momentum in the IT, where only part of the OS is virtualized, allowing more efficiency and lower latency through a reduced overhead [13]. Virtualization offers numerous advantages, starting with the ease of deployment, the more economically efficient use of physical hardware, and the improved security enabled by the isolation which comes along with virtualization. The following will give a brief introduction to the technologies of VMs and containers and discuss their respective implications. Note that further, less common virtualization technologies, e.g. micro-virtualization [14], exist but are not viable for the virtualization in the industrial context.

\section{A. Virtual machine (VM)}

Hardware virtualization software, namely hypervisor or virtual machine monitor (VMM) have first been popular in the 1970ies, when it was allowing multiple tenants to share expensive hardware. The hypervisor may run as an application on top of a running host OS or run on bare-metal with a custom kernel providing a higher performance. On top of the hypervisor multiple guest VMs can run in parallel, whereby each VM can have a different OS and virtual hardware. The hypervisor translates virtual central processing unit (CPU) instructions to the underlying hardware. To enforce the VM isolation, privileged instructions are intercepted and their effect is emulated on the virtual hardware. This instruction set virtualization can be achieved completely in software where the complete instruction stream is just-in-time translated, or in hardware, in case the CPU supports virtualization, where the CPU may take over the execution of the VM instructions, until a privileged instruction is tried to be executed, which is then handed over to the hypervisor [15]. Similarly, memory virtualization can be achieved either in 
software or in hardware. Modern OSs already isolate processes by default through assigning virtual memory.

\section{B. Container}

The term container refers to a virtualized environment on OS-level which offers similar advantages to VMs regarding isolation and ease of deployment while costing significantly less resources, as multiple isolated containers can run directly on the underlying OS and physical or virtual hardware simultaneously with other containers [16]. As can be seen in Fig. 2. an application and its dependencies can be packed into a container, which is running on a container management software. The user-interface for containers is provided by the container manager, offering functionality for building, managing, sharing and running containers [17]. The system independence and easy deployment along with container orchestration software, such as Docker Swarm or Kubernetes (K8s), allows to automate the management, deployment and scaling of containerized applications and is therefore popular for running applications in a cloudcomputing infrastructure [16].

In summary, containers offer an efficient and lightweight virtualization and allow an easy and scalable platform independent deployment of server applications and bring up many novel automation tools assisting the operation, but introduce complexity and overhead compared to bare-metal, allow only Linux as a guest system, and lack support for a graphical user interface (GUI) which some applications may require.

\section{Summary}

To conclude, containers can compete with VMs by providing similar features with better performance. Still VMs will not disappear as they provide functionalities containers can not such as hosting guests with different OS kernels, offering a better isolation, in spite of which VM escapes existing, and supporting applications with GUIs. While these functionalities are not relevant for most cloud services, the current practice tend towards containers running on virtualized infrastructure, inside of VMs. In the long-term containers running on bare-metal are completely capable of replacing VMs in cloud infrastructure, also from a security and privacy perspective. Therefore, a virtualization of industrial automation systems based on container technology is well suited for industrial applications. Boettiger motivates the usage of Docker to enable reproducible and reusable research [18].

\section{Virtualization In Future IndUSTRIAL Automation SySTEMS}

This section investigates how Docker containers can be used for industrial applications and compares performance benchmarks for specific configurations. First, the computational overhead associated with the use of containers is determined (Sec. $\mathrm{V}$-A. Therefore, we compare the determinism of an application running identically in a container, in a VM, and on a baremetal server, based on different execution intervals. In addition, Sec. V-B evaluates the different networking configurations of a Docker container, and compares several characteristics with VMs and bare-metal. For all investigations we have used the equipment listed in Tab. I]
Table I

HARDWARE CONFIGURATIONS

\begin{tabular}{|c|c|l|}
\hline Equipment & QTY & Specification \\
\hline Mini PC & 2 & $\begin{array}{l}\text { Intel Core i7-8809G, 2x16 GB DDR4, } \\
\text { Intel i210-AT \& i219-LM Gibgabit } \\
\end{array}$ \\
& & $\begin{array}{l}\text { NICs, Ubuntu 18.04 LTS 64-bit, } \\
\text { Linux 4.19.103-rt42 }\end{array}$ \\
\hline Switch & 1 & 8-Port Gigabit Ethernet Switch \\
\hline
\end{tabular}

\section{A. Computation Performance}

This section examines the use of a general purpose server for industrial applications. Here, latency and determinism are the main candidates to be analyzed. Therefore, we use Cyclictes ${ }^{1}$ a well-known micro-benchmarking software for the verification of RT characteristics for x86 and x64-based systems and is also the basis of the investigations in [7], [8]. It determines the jitter of a periodically executed task. In order to determine the performance overhead of container and VM virtualization compared to bare-metal the Cyclictest programm is executed on one of the mini PCs listed in Tab. I] We decided to use mini PCs because they are comparable to typical industrial PLCs (e.g. S7-300 [19] and its successor S7-1500 [20]) in terms of space consumption and pricing. Therefore, we ran the following command, where $-\mathrm{N}$ prints the result in nanoseconds, $-\mathrm{n}$ uses clock_nanosleep, $-\mathrm{p}$ sets the RT priority, $-\mathrm{D}$ denotes the duration of the test, and $-i$ sets the interval of the execution: cyclictest $-N-n-p 99-D 10 m-i I$. Since the dependency of Cyclictest on the basis of the priorities was already part of the investigations in [8], we have set the priority to 99 , representing the highest level. In addition, the behavior for different intervals should be investigated, corresponding to the cyclic execution of industrial applications. Based on the requirements of the use cases in Sec. III we will test the behavior for intervals of $1 \mathrm{~ms}, 100 \mu \mathrm{s}, 10 \mu \mathrm{s}$, and $1 \mu \mathrm{s}$. The duration of each test run is set to 10 minutes. In addition, each test run was performed for the following setups:

- No preemption: in this configuration, the Cyclictest software module runs directly on the generic Linux kernel that was supplied with Ubtunu 18.04 LTS.

- Preemption: in this mode, a RT patch for the Linux kernel 4.19. was applied [21] and the "Fully Preemptible" mode has been activated [22].

- Container: in this setup, Cyclictest runs inside a Docker container that has been launched with the - privileged=true flag.

- Virtual machine: the host PC maintains a kernel-based virtual machine (KVM), that is also running on Ubuntu 18.04 LTS in combination with the above mentioned Linux kernel in combination with the RT patch.

For the graphical interpretation of the results, box plots containing 60,000 samples per data series have been created (see Fig. 3).

\footnotetext{
${ }^{1}$ For further information and download see the following website: https://wiki.linuxfoundation.org/realtime/documentation/howto/tools/cyclictest/
} 


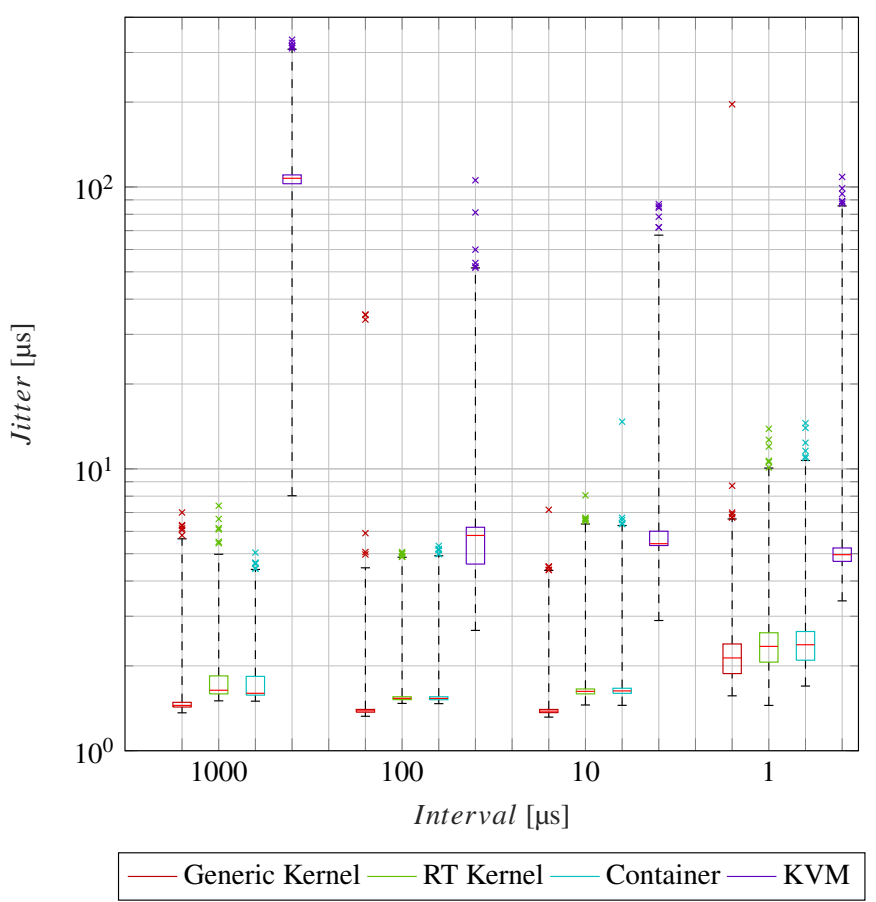

Figure 3. Readings for the Cyclictest software module running on different platforms and and at different intervals

By default, $50 \%$ of the values are located inside the boxes and the outliers are those values that exceed $\pm 1.5 \times I Q R$. Assuming that every second value is close to the median value and the next value is significantly higher, this could lead to the worst case scenario where no values are placed between the boxes and the outliers and therefore only $50 \%$ of the samples lie within the box plots. Since this or similar cases are not sufficient for the rigorous demands of industrial applications, we have selected the outliers so that $99.99 \%$ of all data points are located within the box plots.

It turns out that the timing of the VM is significantly worse than the timing of the other systems. Especially the interval of $1 \mathrm{~ms}$ shows that the median value is 10 times higher compared to the others. The median value decreases with higher processor load, but there are still many outliers in the range of $10-100 \mu \mathrm{s}$. This confirms the previous investigations, which have shown that VMs are not suitable for low-latency RT applications due to the overhead by the additional software stack [13], [23].

Looking at the system with the generic kernel, it can be seen that the median value of this system is the lowest, i.e. the majority of the recorded data values is below that of the other systems. However, if the processor load is increased by reducing the interval, there are some outliers upwards without the use of preemption. This corresponds to the assumption that generic kernels are generally more efficient, but cannot guarantee the level of determinism expected by industrial applications [24].

Next, the RT kernel running on the bare-metal server and the deployment of a container should be compared. Here, the investigations show that there are no significant limitations when using Docker for OS-level virtualization. In an interval of $1 \mathrm{~ms}$ the readings are even slightly lower when running the container versus using preemption, but the reason for this is probably the number of samples. Looking at the other intervals, there is a performance difference of $<0.05 \mu$ s in the median values and $<1 \mu$ s for the maximum values. This means, there is no significant performance impact by using a Docker container. These results comply with the findings in [7], [8].

Another point to consider is the required determinism. If, for example, a reliability of $99.99 \%$, i.e. a failure of $0.01 \%$ of all values, is sufficient for an application, the use of a generic kernel can be advantageous as it brings a higher overall efficiency. In this case, a generic kernel can achieve a jitter of $<7 \mu$ s with a reliability of $99.99 \%$, while an RT kernel is only able to provide about $<10 \mu$ s.

\section{B. Networking}

The next step is to check the network performance of Docker containers for different configurations of the network interface. Furthermore, we assume the following two scenarios:

1) Host-only communication

2) Inter-host communication

In the first scenario, the host-only communication, there are two applications on a single host server that interact with each other. This scenario is useful if an industrial automation system needs to be reconfigured. If, for example, an update of the configuration, firmware or software is required, it is preferable to have both applications on the same system so that the migration between the old and the new application can be triggered without delay by an additional communication network. In cases, where a resilient operation of industrial automation systems is required, however, a redundant instance of the industrial automation system should be placed on a separate host in order to maintain operation of the production line in case of breakdown of the host server. This situation requires inter-host communication.

To enable one of the major advantages of virtualization technologies an automated deployment of industrial automation systems on multiple hosts should be possible. Various orchestration tools are available for a massive and automated container deployment. In this context, K8s and Docker Swarm are the most prominent candidates. Since K8s works on the basis of proxy servers on IP level and a plain L2 communication is not envisaged [25], the investigations have been done on the basis of Docker Swarm. Various settings can be made when configuring Docker containers and using Docker Swarm. Of particular interest here are the network drivers. These vary depending on configuration effort, accessibility, scalability, security level, and performance. All supported network drivers are listed below:

- None: for this container, networking is disabled. Communication outside the container is not possible. Therefore this network driver is not considered further.

- Host: in this configuration all network isolation is deactivated. All containers have direct access to the interfaces of the host and can communicate with each other as well as with external devices.

- Bridge: the default network driver. If no driver is specified when starting a container, it is assigned to this network type. All containers on the host are connected by a network 
bridge and can communicate. However, inter-host communication is not foreseen by the default bridge network. This driver is mainly used for standalone containers.

- Macvlan: macvlan networks allow the assignment of MAC addresses to containers so that they appear as physical devices on the network. The Docker daemon forwards the traffic to containers based on their MAC addresses. This allows containers that use the macvlan driver to avoid the routing through the network stack of the Docker host.

- Ipvlan: ipvlan can be compared with the network driver macvlan and offers a L2 and L3 mode. In contrast to the network driver macvlan, the endpoints using ipvlan have the same MAC address.

- Overlay: overlay networks connect the Docker daemons of multiple hosts and enable the container-to-container communication of multiple hosts. By using overlay networks, the routing of packets is handled by Docker and makes routing at OS-level obsolete. For this reason this is the default network driver for Docker Swarm services.

As already described in Sec. III the requirements for industrial use cases are usually expressed by the cycle time, which consists of the time to exchange the messages and the computing time of the algorithm. Since this time includes both the reception of the sensor values and the transmission of the control outputs to the actuators, the round-trip time (RTT) for the respective network drivers is determined and compared with bare-metal and VMs. Once more, box plots with 60,000 data samples per series were generated for the evaluation of these measurements. Again, the outliers were defined in such a way that $99.99 \%$ of all values are within the outer limits of the box plot.

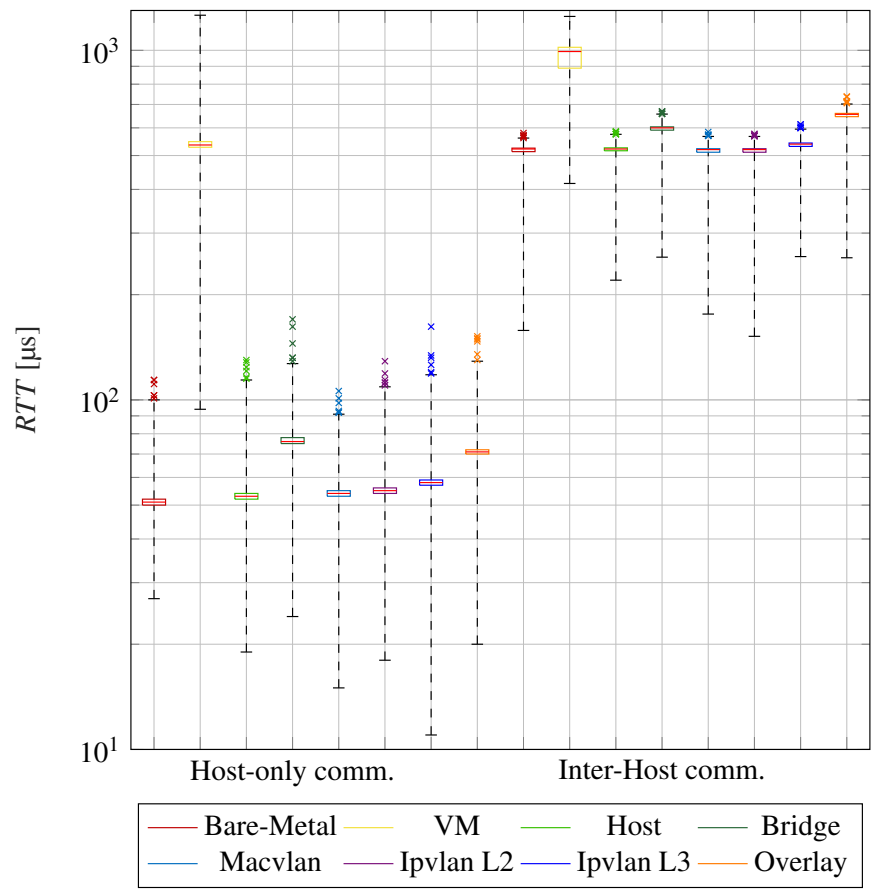

Figure 4. Readings for the measurement of the RTT for for host-only and interhost communication

As shown in Fig. 4. VMs perform significantly worse than the other configurations in both host-only and inter-host tests.
In host-only communication, the median value for the RTT is about 10 times higher than in all other network configurations. In addition, the median values for the host, macvlan and ipvlan configuration differ from the bare-metal system by only $2 \mu \mathrm{s}$ and $3 \mu$ s for the host-only measurements and are equal for interhost communication. The maximum values for these network configurations also only vary in the range of $582 \mu \mathrm{s} \pm 5 \mu$ s. This means that selecting these network drivers for the deployment of a Docker container will not degrade network performance compared to the use of bare-metal.

Next, looking at the standard drivers for standalone containers and Docker Swarm clusters using bridge and overlay modes, it can be seen that these are about $20 \%$ slower for both of the investigated scenarios. From a performance point of view, the network drivers host, macvlan and ipvlan are preferable, as they have a very low overhead and therefore no significant effect regarding the RTT of the communication system.

In addition to performance, other criterions have to be considered when selecting a suitable virtualization method. These are the configuration complexity for setting up the respective system (configuration), which network interfaces are required (networking), whether automated deployment of multiple instances is possible (scalability), and the system's vulnerability (security level), where a threat model for attacker types A, B, C, and D was introduced in Sec. IIII Therefore, Tab. III sums up the key aspects for each network configuration.

First the bare-metal should be evaluated. Here the performance is fine and the applications can directly access the physical network adapter of the system. Furthermore, this system allows L2 communication that is required by applications that belong to RT class C. However, automated provisioning and configuration is not possible and there is no isolation, so security concerns are an important factor when using the infrastructure without virtualization. In terms of network performance and isolation, VMs behave in the opposite way compared to baremetal. Performance is significantly worse compared to all other systems analyzed, but the security level is higher due to isolation when using a fully virtualized system. Therefore, VMs can only be used if performance is not the primary focus.

Next, the different Docker network configurations should be discussed. In host mode the containers network can be accessed directly over the host's adapter, exposing it to everything connected to the host and is therefore comparable to bare-metal. In terms of performance there is a small overhead given by the virtualization, but there is no scalability, since this network driver cannot be used for a Swarm service. In addition, attackers $\mathrm{D}$ and $\mathrm{C}$ can connect to the application if it is not authenticated and attackers $\mathrm{A}$ and $\mathrm{B}$ can monitor any traffic going over the host adapter, as host mode effectively lifts the networking isolation Docker offers by default. From a security perspective, this mode should only be used when necessary and with trusted services running on the container.

A Bridge network where the container connects to the host network over a virtual bridge is enabled by default. Nonetheless the official Docker documentation discourages from using default bridges, arguing that every unrelated container without a specified network communicates over the same bridge and may perform ARP spoofing or MAC flooding attacks. Assuming 
Table II

COMPARISON OF THE DIFFERENT NETWORK CONFIGURATIONS

\begin{tabular}{|c|c|c|c|c|c|c|c|c|c|}
\hline \multirow{2}{*}{$\begin{array}{l}\text { Network } \\
\text { Config. }\end{array}$} & \multicolumn{2}{|c|}{ Config. of Host / Docker } & \multicolumn{2}{|c|}{ Networking } & \multirow[t]{2}{*}{ Scalability } & \multicolumn{2}{|c|}{ Security Level } & \multicolumn{2}{|c|}{ Performance (RTT) } \\
\hline & Host & Docker & $L 2$ & $L 3$ & & $\begin{array}{l}\text { Guest/Host } \\
\text { isolation }\end{array}$ & $\begin{array}{l}\text { Network } \\
\text { isolation }\end{array}$ & $\begin{array}{l}\text { Host- } \\
\text { only }\end{array}$ & $\begin{array}{l}\text { Inter- } \\
\text { Host }\end{array}$ \\
\hline $\begin{array}{l}\text { Bare- } \\
\text { Metal }\end{array}$ & Not required & - & Yes & Yes & No & - & No & $51 \mu \mathrm{s}$ & $522 \mu \mathrm{s}$ \\
\hline$V M$ & $\begin{array}{l}\text { Config. of a } \\
\text { Linux bridge } \\
\text { per host }\end{array}$ & - & Yes & Yes & Yes & Yes & No & $536 \mu \mathrm{s}$ & $992 \mu \mathrm{s}$ \\
\hline Host & Not required & Not required & Yes & Yes & No & No & No & $53 \mu \mathrm{s}$ & $522 \mu \mathrm{s}$ \\
\hline Bridge & $\begin{array}{l}\text { Config. of a } \\
\text { Linux bridge } \\
\text { per host }\end{array}$ & $\begin{array}{ll}\text { Config. } & \text { of } \\
\text { Docker } & \\
\text { network } & \end{array}$ & Yes & Yes & $\begin{array}{l}\text { Yes, but addi- } \\
\text { tional config. } \\
\text { per Host }\end{array}$ & No & Yes & $76 \mu \mathrm{s}$ & $600 \mu \mathrm{s}$ \\
\hline Macvlan & $\begin{array}{l}\text { Only for Host- } \\
\text { to-Container } \\
\text { comm. }\end{array}$ & $\begin{array}{ll}\text { Config. of } \\
\text { Docker } \\
\text { network }\end{array}$ & Yes & Yes & $\begin{array}{l}\text { Yes, but addi- } \\
\text { tional config. } \\
\text { per Host }\end{array}$ & Yes & No & $54 \mu \mathrm{s}$ & $520 \mu \mathrm{s}$ \\
\hline $\begin{array}{l}\text { Ipvlan } \\
\text { (L2) }\end{array}$ & Not required & $\begin{array}{l}\text { Config. of } \\
\text { Docker } \\
\text { network }\end{array}$ & No & Yes & $\begin{array}{l}\text { Yes, but addi- } \\
\text { tional config. } \\
\text { per Host }\end{array}$ & Yes & No & $55 \mu \mathrm{s}$ & $520 \mu \mathrm{s}$ \\
\hline $\begin{array}{l}\text { Ipvlan } \\
\text { (L3) }\end{array}$ & Not required & $\begin{array}{l}\text { Config. of } \\
\text { Docker } \\
\text { network }\end{array}$ & No & Yes & $\begin{array}{l}\text { Yes, but addi- } \\
\text { tional config. } \\
\text { per Host }\end{array}$ & Yes & No & $58 \mu \mathrm{s}$ & $539 \mu \mathrm{s}$ \\
\hline Overlay & Not required & Not required & No & Yes & Yes & Yes & Yes & $71 \mu \mathrm{s}$ & $656 \mu \mathrm{s}$ \\
\hline
\end{tabular}

an isolated user-defined bridge is used, attacker B can only communicate to the host and over the specified ports, attacker A has full access to the bridge network. Attacker C and D only have access to the bridge network over ports forwarded by the host interface. User-defined bridges also provide a Swarm mode.

As macvlan makes the container appear as a physical device with an own MAC address, it is well suited for industrial communication. While a firewall can still restrict access by attacker $\mathrm{D}$, attacker $\mathrm{C}$ can communicate with the device because the macvlan must be on the same subnet as the host interface. The traffic between the container and the underlying hosts is filtered by kernel modules to provide isolation, therefore access for attacker type A is restricted. Attackers of type B are isolated from the host but can access other containers macvlan network. If access by the host should not be allowed, configuration of the host is not required. For the use in a Docker Swarm cluster, a configuration on Docker level is also required for this network driver once per host.

Ipvlan L2 mode is functionally equivalent to the macvlan bridge mode, with the difference that all containers running on the same host are assigned the same MAC address. This may be useful when it is not possible to run the interface in promiscuous mode, which would be required for macvlan. In addition, certain network switches have a security feature where a physical port only transmits packets from one known MAC address to mitigate adress resolution protocol (ARP) spoofing attacks. In this case ipvlan is preferred over macvlan as all containers have the same MAC address and can share the secure port. Also, for whitelisting base MAC filters ipvlan may be preferred. Ipvlan L3 mode different subnets and networks can communicate when the parent interface is the same. Like in macvlan, direct commu- nication with parent interface is not possible. In ipvlan L3 mode interfaces do not allow broadcast or multicast traffic, these are handled by the host. Routing, in contrary to L 2 mode, is done by the parent interface according to the namespace, therefore L3 mode is better suited for non-trusted environments [26]. If an application requires L2 communication, and the security policies allow the assignment of a physical MAC address for each container, the macvlan network driver should be selected.

Overlay networks are the default network driver when deploying a Docker Swarm service and allow multiple Docker hosts to connect to a virtual network on top of an existing network. Therefore, the Docker daemons of each host are configured automatically This avoids misconfiguration, but causes an overhead that results in a loss of performance compared to the host, macvlan and ipvlan network drivers. By default, the traffic is not encrypted and can be seen by attacker type A, B, and C. Attacker D could also see unencrypted overlay traffic if two Docker daemons are connected over the internet and passes attacker D. Encryption can be easily enabled and would restrict attacker $\mathrm{C}$ and $\mathrm{D}$ from viewing the data.

\section{Summary}

The investigations have shown that the use of containers leads to a better performance compared to VMs in addition to the increased efficiency. Furthermore, container-based applications perform only slightly worse than bare-metal applications and can be used for industrial applications of each of the proposed RT classes. A look at the computation performance shows that a Linux RT kernel in combination with Docker can reliably run the cyclic execution of applications in intervals of $1 \mu \mathrm{s}-1 \mathrm{~ms}$ with a jitter of $<15 \mu$ s. Comparing this with the RT kernel without 
using Docker, the maximum jitter is about $1 \mu$ s lower, which is almost negligible. From a security perspective, traffic encryption is recommended, but the non-negligible performance penalty does not allow network encryption for time-critical applications, then security must be achieved through a high degree of traffic isolation and other security measures. Looking deeper into the networking of each technology, we concluded that the macvlan network driver is best suited for applications that have high latency requirements and require L2 and L3 communications, while the overlay driver should be used when a higher level of security needs to be achieved and the application can handle RTTs that are $20 \%$ higher than those of other configurations.

\section{CONCLUSION}

In this paper, we have examined the possibilities of virtualization in the industrial landscape. Performance comparisons for bare-metal applications, VMs and containers were conducted and evaluated for industrial needs. Furthermore, the available network drivers of Docker containers were listed and compared based on configuration effort, accessibility, scalability, security level, and performance. Again, the performance for VMs and applications without virtualization technology was compared. The analyses indicate that the flexibility gained by virtualization can be achieved for industrial applications without violating the stringent RT requirements in the industrial landscape, if Docker containers with a suitable configuration are used.

\section{REFERENCES}

[1] P. Gaj, J. Jasperneite, and M. Felser, "Computer Communication Within Industrial Distributed Environment-a Survey," IEEE Transactions on Industrial Informatics, vol. 9, no. 1, pp. 182-189, Feb. 2013. DOI: 10 . 1109/TII.2012.2209668

[2] E. Sisinni, A. Saifullah, S. Han, U. Jennehag, and M. Gidlund, "Industrial Internet of Things: Challenges, Opportunities, and Directions," IEEE Transactions on Industrial Informatics, vol. 14, no. 11, pp. 4724-4734, Nov. 2018. DOI: 10.1109/TII.2018.2852491

[3] M. Gundall, J. Schneider, H. D. Schotten, M. Aleksy, D. Schulz, N. Franchi, N. Schwarzenberg, C. Markwart, R. Halfmann, P. Rost, D. Wübben, A. Neumann, M. Düngen, T. Neugebauer, R. Blunk, M. Kus, and J. Grießbach, " $5 \mathrm{G}$ as Enabler for Industrie 4.0 Use Cases: Challenges and Concepts," in 2018 IEEE 23rd International Conference on Emerg ing Technologies and Factory Automation (ETFA), vol. 1, Sep. 2018, pp. 1401-1408. DOI: 10.1109/ETFA.2018.8502649

[4] Y. Mao, C. You, J. Zhang, K. Huang, and K. B. Letaief, "Mobile Edge Computing: Survey and Research Outlook," ArXiv, vol. abs/1701.01090, 2017.

[5] S. Tayade, P. Rost, A. Maeder, and H. D. Schotten, "Device-Centric Energy Optimization for Edge Cloud Offloading," in GLOBECOM 2017 - 2017 IEEE Global Communications Conference, Dec. 2017, pp. 1-7. DOI: 10.1109/GLOCOM.2017.8254628

[6] O. Givehchi, J. Imtiaz, H. Trsek, and J. Jasperneite, "Control-as-a-service from the cloud: A case study for using virtualized PLCs," in 2014 10th IEEE Workshop on Factory Communication Systems (WFCS 2014), May 2014, pp. 1-4. DOI: 10.1109/WFCS.2014.6837587

[7] T. Goldschmidt, S. Hauck-Stattelmann, S. Malakuti, and S. Grüner, "Container-based architecture for flexible industrial control applications," Journal of Systems Architecture, vol. 84, pp. 28-36, 2018.
[8] A. Moga, T. Sivanthi, and C. Franke, "OS-Level Virtualization for Industrial Automation Systems: Are We There Yet?" In Proceedings of the 31st Annual ACM Symposium on Applied Computing, ser. SAC '16, Pisa, Italy: Association for Computing Machinery, 2016, pp. 1838-1843. DOI: 10.1145/2851613.2851737 [Online]. Available: https://doi.org/10. $1145 / 2851613.2851737$

[9] M. Wollschlaeger, T. Sauter, and J. Jasperneite, "The Future of Industrial Communication: Automation Networks in the Era of the Internet of Things and Industry 4.0," 1, vol. 11, Mar. 2017, pp. 17-27. DOI: 10. 1109/MIE.2017.2649104

[10] J. Pfrommer, A. Ebner, S. Ravikumar, and B. Karunakaran, "Open Source OPC UA PubSub over TSN for Realtime Industrial Communication,” Jul. 2018. DOI: 10.1109/ETFA.2018.8502479

[11] C. Mannweiler, B. Gajic, P. Rost, R. S. Ganesan, C. Markwart, R. Halfmann, J. Gebert, and A. Wich, "Reliable and Deterministic Mobile Communications for Industry 4.0: Key Challenges and Solutions for the Integration of the 3GPP 5G System with IEEE," in Mobile Communication - Technologies and Applications; 24. ITG-Symposium, May 2019, pp. 1-6.

[12] B. Zhu, A. Joseph, and S. Sastry, "ATaxonomy of Cyber Attacks on SCADA Systems,” pp. 380-388, 2011.

[13] W. Felter, A. Ferreira, R. Rajamony, and J. Rubio, "An updated performance comparison of virtual machines and Linux containers," in 2015 IEEE International Symposium on Performance Analysis of Systems and Software (ISPASS), Mar. 2015, pp. 171-172. DOI: 10.1109/ISPASS 2015.7095802

[14] Bromium, "Whitepaper: Micro-virtualization vs Software Sandboxing," 2013.

[15] R. Uhlig, G. Neiger, D. Rodgers, A. L. Santoni, F. C. Martins, A. V Anderson, S. M. Bennett, A. Kagi, F. H. Leung, and L. Smith, "Intel virtualization technology," Computer, vol. 38, no. 5, pp. 48-56, 2005.

[16] L. Yin, J. Luo, and H. Luo, "Tasks Scheduling and Resource Allocation in Fog Computing Based on Containers for Smart Manufacturing," IEEE Transactions on Industrial Informatics, vol. 14, no. 10, pp. 4712-4721, Oct. 2018. DOI: 10.1109/TII.2018.2851241

[17] T. Combe, A. Martin, and R. Di Pietro, "To Docker or Not to Docker: A Security Perspective," IEEE Cloud Computing, vol. 3, no. 5, pp. 54-62, 2016. DOI: 10.1109/MCC.2016.100

[18] C. Boettiger, "An Introduction to Docker for Reproducible Research," SIGOPS Oper. Syst. Rev., vol. 49, no. 1, pp. 71-79, Jan. 2015. DOI: 10.1145/2723872.2723882 [Online]. Available: https://doi.org/10.1145/ 2723872.2723882

[19] S. AG, "SIMATIC S7-300C: Die kompakten Controller für die Fertigungsautomatisierung bieten vielfältige Innovationen und integrierte Funktionen,” 2011. [Online]. Available: https://c4b.gss.siemens.com/ resources/images/articles/e20001-a760-p210.pdf

[20] "Delivery Release for the new SIMATIC S7-1500 Controllers," Mar. 2013. [Online]. Available: https://support.industry.siemens.com/cs/ document/67856446/

[21] V. Yodaiken and M. Barabanov, "Real-time linux applications and design,"

[22] S.-T. Dietrich and D. Walker, "The evolution of real-time linux," in 7th RTL Workshop, Citeseer, 2005.

[23] A. M. Joy, "Performance comparison between Linux containers and virtual machines," in 2015 International Conference on Advances in Computer Engineering and Applications, 2015, pp. 342-346.

[24] L. Abeni, A. Goel, C. Krasic, J. Snow, and J. Walpole, "Ameasurementbased analysis of the real-time performance of linux," in Proceedings. Eighth IEEE Real-Time and Embedded Technology and Applications Symposium, 2002, pp. 133-142.

[25] V. Marmol, R. Jnagal, and T. Hockin, "Networking in containers and container clusters," Proceedings of netdev 0.1, 2015.

[26] M. Bandewar and E. Dumazet, "IPVLAN - The beginning," in Proceedings of netdev 0.1, Feb. 2015. 